\title{
An ubiquitous smart guitar system for collaborative musical practice
}

\author{
Luca Turchet ${ }^{\mathrm{a}}$ and Mathieu Barthet ${ }^{\mathrm{b}}$

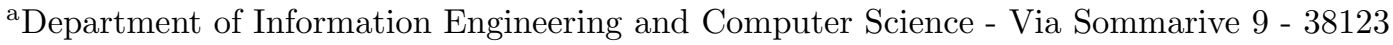 \\ Povo (Trento, Italy); ${ }^{\mathrm{b}}$ Centre for Digital Music, School of Electronic Engineering and \\ Computer Science, Queen Mary University of London, Mile End Road, London E1 4NS \\ (United Kingdom)
}

\section{ARTICLE HISTORY}

Compiled June 24, 2019

\begin{abstract}
Ubiquitous music (UbiMus) proposes to study how social interaction with mobile and distributed technologies can converge to form novel creativity support tools and music artistic practices. A recent field overlapping with UbiMus is the Internet of Musical Things, which refers to ecosystems of interconnected embedded computers (Musical Things) enabling users to produce, interact with or experience musical content. Musical Things embed electronics, sensors, data forwarding and processing software into physical or virtual objects. Smart musical instruments (SMIs) are an emerging class of Musical Things provided with capabilities of capturing and receiving data supporting instrumental musical practice. Due to their portability and self-containdeness, SMIs enable novel ubiquitous interactions between performers of acoustic and digital musical instruments. After a review of current trends in SMI research, we propose an ubiquitous smart guitar system which uses the guitar as a hub for collaborative music making. We then present a survey conducted with 18 performers to assess the usability, creativity support and engagement with the system. Results show a positive emotional engagement with the system which overall was found easy to use and novel. We also discuss several barriers to creative interaction related to the size of the user interface, creative agency and personalisation.
\end{abstract}

\section{KEYWORDS}

Smart musical instruments; Internet of Musical Things; Ubiquitous music; Smart guitar; Networked music performance; Usability; Creativity support

\section{Introduction}

Ubiquitous music (UbiMus) is a multidisciplinary research field which lies at the intersection of sound and music computing, human-computer interaction and music cognition amongst other disciplines (Keller, Lazzarini, \& Pimenta, 2014). It proposes to design and study complex systems of human agents, material resources (objects and sonic cues), and creativity support tools based on mobile and distributed technologies (Keller \& Lazzarini, 2018). The computational perspective in UbiMus targets the development of computational tools leveraging ubiquitous computing concepts and technology (Satyanarayanan, 2001; Weiser, 1991), while other approaches focus on educational, philosophical or creativity issues (Keller \& Lazzarini, 2018). A recent field overlapping with UbiMus is the Internet of Musical Things (IoMusT) (Turchet, Fis- 
chione, Essl, Keller, \& Barthet, 2018). Besides UbiMus, the IoMusT originates from the integration of many lines of existing research including the Internet of Things (Borgia, 2014), new interfaces for musical expression (NIME) (Jensenius \& Lyons, 2017), networked music performance systems (Rottondi, Chafe, Allocchio, \& Sarti, 2016), music information retrieval (Burgoyne, Fujinaga, \& Downie, 2016), participatory music (Wu, Zhang, Bryan-Kinns, \& Barthet, 2017), and human-computer interaction (HCI) (Rowland, Goodman, Charlier, Light, \& Lui, 2015).

Considering a computer science perspective, in (Turchet, Fischione, et al., 2018), the authors defined a Musical Thing as "a computing device capable of sensing, acquiring, processing, or actuating, and exchanging data serving a musical purpose" and the IoMusT as "the ensemble of interfaces, protocols and representations of musicrelated information that enable services and applications serving a musical purpose based on interactions between humans and Musical Things or between Musical Things themselves, in physical and/or digital realms. Music-related information refers to data sensed and processed by a Musical Thing, and/or exchanged with a human or with another Musical Thing". Proposing a theoretical framework for UbiMus, Keller and Lazzarini discussed a vision of the IoMusT, where the IoMusT is seen as part of ubiquitous music ecosystems (Keller \& Lazzarini, 2017), which function as technological hubs supporting the integration of audio and interaction tools Keller and Lazzarini (2018).

The IoMusT technological infrastructure enables the development of ecosystems of interoperable devices that connect musicians with one another, as well as with audiences. This multiplies the interaction possibilities between e.g., performers, composers, conductors, studio producers, live sound engineers, and audience members, both in colocated and remote settings. One of the building blocks of the IoMusT paradigm are the so-called "smart musical instruments (SMIs)", an emerging class of musical instruments characterised by embedded sensors, actuators, wireless connectivity, and on-board processing (Turchet, 2019). The domains of applications of SMIs go beyond traditional professional music settings such as studios and stages, as they embed technologies for sound production and processing. To this end, an investigation of SMIs in the context of ubiquitous music falls within the remit of the third HCI wave investigating technology which spread from the workplace to home and everyday life and culture (Bødker, 2015).

The relationship between SMIs and UbiMus has thus far not been thoroughly investigated by the NIME or UbiMus research communities. SMIs have the potential to enable musical interactions between acoustic and digital instrument performers leveraging ubiquitous technologies and to involve audiences or non musicians in creative processes. This paper, which extends the study reported in (Turchet \& Barthet, 2018b), aims to better understand how the novel class of SMIs can support ubiquitous music. We first present current trends in SMI research and describe the main principles of SMIs. We then propose an ubiquitous smart guitar system composed of an acoustic guitar with a smart add-on and a smartphone app for collaborative music making. We present results from an evaluation conducted with 18 participants and discuss its usability, creativity support and overall experience in the context of ubiquitous musical activities. 


\section{Instances of smart musical instruments}

SMIs result from the integration of a variety of technologies and concepts such as sensor- and actuator-based "augmented instruments" (Miranda \& Wanderley, 2006; Turchet, 2018b) (e.g., McPherson (2015); Overholt, Berdahl, and Hamilton (2011)), embedded acoustic and electronic instruments (Berdahl, 2014; MacConnell et al., 2013), networked music performance (Rottondi et al., 2016), Internet of Things (Borgia, 2014), as well as methods for sensor fusion (Pardue, Harte, \& McPherson, 2015), audio pattern recognition (Dannenberg \& Hu, 2003), semantic audio (Slaney, 2002), and machine learning (Fiebrink \& Caramiaux, 2016).

An example of SMI is the Sensus Smart Guitar developed by MIND Music Labs ${ }^{1}$. It consists of a hollow body guitar augmented with several sensors embedded in various parts of the instrument, on-board processing, a system of multiple actuators attached to the soundboard, and interoperable wireless communication (using state-of-the art protocols for wireless transmission and reception such as Wi-Fi and Bluetooth, as well as for exchange of musical data such as Musical Instruments Digital Interface [MIDI] and Open Sound Control [OSC]). The internal sound engine is based on the ELK music operating system ${ }^{2}$, affords a large variety of sound effects and sound generators, and is programmable via dedicated apps on desktop PCs, smartphones, and tablets.

A second instance of SMI, which has been developed within the context of academic research, is the Smart Cajón described in (Turchet, McPherson, \& Barthet, 2018a) and (Turchet, McPherson, \& Barthet, 2018b). This instrument consists of a conventional acoustic cajón augmented with sensors, Wi-Fi connectivity and motors for vibro-tactile feedback. The Bela board is used for low-latency audio and sensors processing (McPherson, Jack, \& Moro, 2016) and runs a sound engine providing sampling and various audio effects. A peculiarity of the instrument's embedded intelligence is the use of sensor fusion and semantic audio techniques to estimate the location of the players' hits on the instrument's front and side panels, and to use the predictions to map different parts of the instrument to sound samples simulating various techniques and/or percussive instruments.

Another example of SMI is the Smart Mandolin reported in (Turchet, 2018a). This is a classic Neapolitan mandolin augmented with different types of sensors, a microphone, a loudspeaker, wireless connectivity to both local networks and the Internet, and a low-latency audio processing board. Various use cases were implemented, which leverage the smart qualities of the instrument. These include the programming of the instrument via applications for smartphones and desktop computer, as well as the wireless control of devices enabling multimodal performances such as screens displaying visuals, smartphones, and tactile devices used by the audience (Turchet \& Barthet, 2019).

\section{Features of SMIs enabling ubiquitous musical activities}

The term ubiquitous music (UbiMus) has been proposed to relate to "practices that empower participants of musical experiences through socially oriented, creativityenhancing tools", leveraging mobile communication and information devices due to their portability, mobility, connectivity and availability (Keller et al., 2014). As stated in Keller and Lazzarini (2018):

\footnotetext{
${ }^{1}$ https://www.mindmusiclabs.com/sensus

${ }^{2}$ https://www.mindmusiclabs.com/ELK
} 
A key challenge in ubimus research is the provision of intuitive tools for diverse creative tasks. Ubimus systems should ideally support the users' creative potential by fostering easy access to material and social resources. Depending on the approach, the development of certain technologies may have the unwanted side-effect of restricting the support to a small user base. This is often the case in the area of new music instruments. For example, while the provision of custom-made, special purpose hardware interfaces, as proposed by the tangible user interface design approaches (Fitzmaurice et al. 1995; Ishii et al. 2001) can fulfil the requirements of transparency and naturalness reducing the cognitive load of complex tasks, they do not guarantee a wide-ranging adoption by a large cohort of users (often due to financial, as well as distribution and maintenance constraints). Ubimus attempts to solve this issue by focusing on repurposing, which entails the use of existing, everyday technology, for creative ends: personal mobile devices (Flores et al. 2010, Lazzarini et al. 2012), web technologies (Lazzarini et al. 2015b), and DIY hardware (Lazzarini et al 2015c).

Traditional acoustical musical instruments are not part of everyday technology and generally restricted to those possessing a certain degree of musical sophistication (Müllensiefen, Gingras, Musil, \& Stewart, 2014). This creates frictions to incorporate them into the design of ubiquitous music systems as it de facto limits their use by non musicians, a category of users central to UbiMus research. Reciprocally, UbiMus interfaces for non musicians may be too restrictive for musicians who are used to instruments which are "not easy to play at first but do afford the development of a high degree of musicality" (Wessel \& Wright, 2002). Our approach is to design interfaces making traditional acoustical musical instruments "compatible" with everyday technologies providing new affordances for creative action in UbiMus settings, for example between musicians and non musicians. We discuss below three SMIs features that can contribute to facilitate UbiMus activities:

SMI self-contained nature. In contrast to other digital music interfaces (DMIs) such as augmented instruments (Miranda \& Wanderley, 2006; Turchet, 2018b), SMIs embed several components in a unique standalone device. Such a self-contained nature provides benefits which could not be obtained with the large amount of equipment otherwise needed to create a similar setup (e.g., by combining a soundcard, cables, microphones, loudspeaker, MIDI controllers, laptop). These benefits include ease of setup, portability, reduction of required space, and freedom of movement which are aspects all deemed important by musicians (Martinez-Avila, Greenhalgh, Hazzard, Benford, \& Chamberlain, 2019; Rossitto et al., 2018). SMIs limit the amount of devices to be connected and powered. SMI musicians benefit from computer music production technologies while simply turning on a ready-to-use SMI which is easy to carry when traveling.

SMI network connectivity. The wireless connectivity options embedded in a SMI enables the transmission and reception of content communicated via local and remote networks. Such connectivity can be used to support collaborative music making from any locations with Internet networks (both with other musicians and audience members) provided network latency does not hinder asynchronous/synchronous musical interactions. As discussed in (Schiavoni, de Faria, \& Manzolli, 2018), different network addressing methods (e.g. unicast, multicast, broadcast) can be leveraged to develop new collaborative music creation and performances. Added value of network connectivity for SMIs can also be found in using ubiquitous resources such as the online audio repositories and services proposed by the Audio Commons Initiative ${ }^{3}$ (Font et al., 2016). Semantic sound objects available through browser-based interfaces (Stolfi,

${ }^{3}$ http: //audiocommons.org 
Ceriani, Turchet, \& Barthet, 2018) could be used in conjunction with SMIs to enable instrumentalists quick access to a large variety of sounds that could be uploaded to their SMIs. Network connectivity also provides the possibility for SMIs to leverage cloud computing (see Turchet, Fischione, et al. (2018) for a discussion). Ubiquitous musical interactions could also be envisioned by connecting SMIs to web-based social networks.

SMI embedded intelligence. The intelligent systems embedded in SMIs can provide useful proactivity and context-awareness capabilities for ubiquitous musical activities. Compared to typical augmented instruments, SMIs switch from being reactive to what the musicians play to being proactive, for example by assisting musicians to take musical decisions (Turchet, 2018b). According to the vision proposed in Turchet (2019), a smart instrument is characterized by five core capabilities that define its embedded intelligence: i) knowledge management, i.e., the capability of maintaining knowledge about itself and the environment; ii) reasoning, i.e., the capability of making inferences on the acquired knowledge; iii) learning, i.e., the capability of learning from previous experience; iv) human-smart instrument interaction, i.e., the capability of interacting with the player in ways that extend the bare sound production, such as adaptation and proactivity; v) smart instrument-Musical Things interaction, i.e., the capability of wirelessly exchanging information with a diverse network of interoperable Musical Things. The following speculative scenarios illustrate how UbiMus activities may benefit from proactivity and context-awareness features of SMIs: 1) Audience-driven set list: a SMI which proposes songs to play to the musician based on the musical tastes of the audience as characterised from Spotify profiles retrieved from smartphones, 2) Audience feedback for performers: a SMI which is aware of the audience's activity, as characterised from inertial measurement unit data from audiences' smartphones, and makes suggestions of tempo or styles of songs to play to the performer, 3) Active audience participation in creative process: a SMI which enables audience members to contribute to the creative music process by generating musical structures or more generally aesthetic narratives in response to intentions interpreted by the SMI.

The smart musical instrument features reviewed above can facilitate various types of human-human and human-machine interactions: interactions between musicians and their instruments, between musicians and audience members or non musicians, and/or between musicians. Such technologically-mediated interactions may occur not only in co-located settings but also remotely thanks to the Internet. Ubiquitous musical activities may be developed leveraging these possibilities.

\section{Examples of use cases of SMIs in ubiquitous musical activities}

This section describes two use cases of SMIs in the context of ubiquitous musical activities. Such use cases represent two distinct scenarios that have been reported in the smart instruments literature.

Smart Musical Instruments as hubs for collaborative music making. SMIs may be equipped with an embedded loudspeaker or a system that mechanically acts on the vibrating components of the instrument which radiate the sound (such as a system of multiple actuators attached on a guitar's soundboard). This feature, coupled with the capabilities of exchanging data with connected Musical Things as well as processing and generating audio signals, enables the ubiquitous use of a SMI as a hub for collaborative music making (such as jam sessions). A connected Musical Thing 


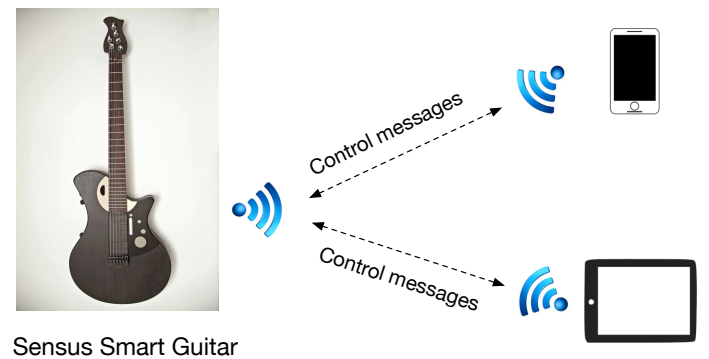

Figure 1. A schematic representation of a jam between three musicians involving the Sensus smart guitar and dedicated apps running on a smartphone and a tablet.

may transmit to the SMI messages that interactively control a sound generator (e.g., synthesizers or drum machines), the sounds of which are reproduced by the SMI itself when the player is playing it. More than one Musical Thing can be connected to the same SMI so that different performers could jam together thanks to a unique SMI.

Such a use case has been implemented with the Sensus Smart Guitar. In (Turchet, Benincaso, \& Fischione, 2017), the authors describe an app running on both Androidand iOS-based smartphones and tablets which enables jamming with the Sensus (see Figure 1). The app allowed participants to wirelessly stream audio content and/or musical messages (via OSC or MIDI) towards the instrument. Such data were fed into the instrument's sound engine and then reproduced by its sound delivery system, while the performer was playing on the instrument. More than one smart device running the app were used simultaneously, which allowed multiple players to take part to the jam session. In turn, the smart guitar player by acting on the instrument's sensor interface could change the behaviour of the app running on one or more smart devices (by changing presets and/or the interface layout).
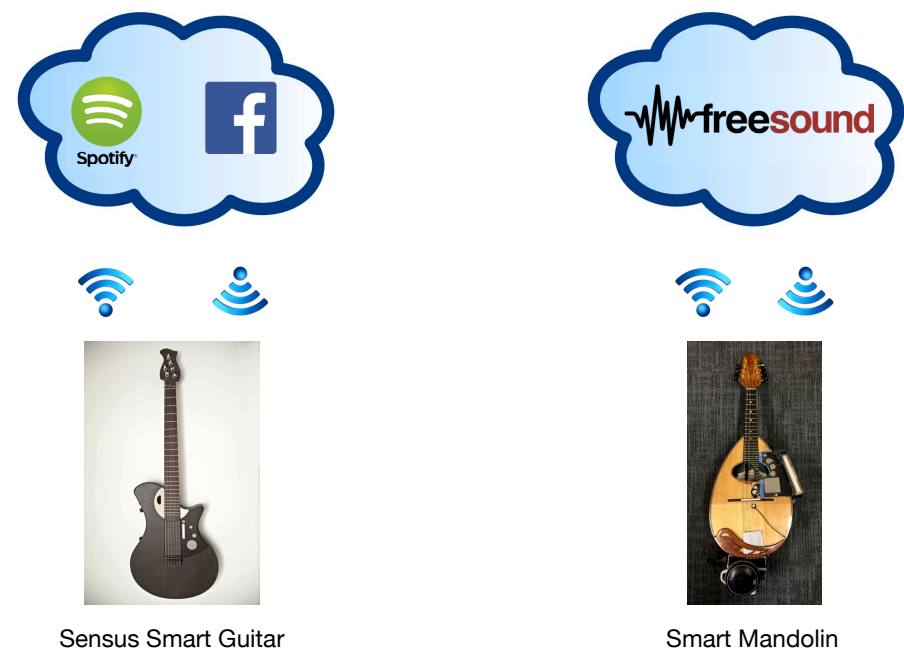

Figure 2. A schematic representation of the interaction between smart musical instruments and the cloud. Each smart musical instrument (Sensus Smart Guitar on the left, Smart Mandolin on the right) is capable of communicating and exchanging online audio information with cloud platforms such as Spotify, Facebook and Freesound.

Cloud-based smart musical instruments interaction. Thanks to their wireless connectivity features, SMIs can receive and reproduce audio signals streamed 
from remote repositories. This may be achieved either via direct connectivity of the instrument to the Internet, or by leveraging another Internet-enabled smart device as a bridge towards the cloud (e.g., a smartphone). This may allow SMI players: (i) to play over downloaded audio content, while reproduced by the instrument (e.g., for improvisation or rehearsing purposes), or (ii) to select sounds that can be used as tones produced by the instrument through sample-based synthesis. The Internet provides access to very large amount of digital audio content, from instrument samples and sound effects, to human- and nature-related environmental sounds and produced songs ready to use in performance. An emerging online community has formed fostering a culture of sharing of creative artefacts (video, audio, photos, etc.). Creative Commons provides a legal framework enabling the reuse and remix of creative artefacts. The Audio Commons Initiative (Font et al., 2016) promotes an ecosystem of interconnected audio content, users (e.g. creators, consumers) and software systems for audio retrieval and processing. Such ecosystem can be of benefit for SMIs leading towards UbiMus activities involving the repurposing of online audio content. Figure 2 provides a conceptual representation of two related use cases which are discussed below.

An example of this use case is reported in (Turchet et al., 2017) to find backing tracks with the Sensus Smart Guitar. An application running on iOS-based smartphones was implemented, which streamed towards the Sensus guitar some songs selected from Spotify via Bluetooth. The smart guitar players could jam on top of the tracks of their favourite artists thanks to the instrument's capability of reproducing (via the actuators attached to the soundboard) both the downloaded audio and the performed guitar sounds. In addition, thanks to recording features accessible through the switch buttons embedded in the instrument, the players were enabled to record their jam and stream the resulting audio file back to the smartphone. Such a file could then be shared on Facebook.

In the same vein, the Smart Mandolin (Turchet, 2018a) has been used in interaction with the Freesound ${ }^{4}$ online audio content repository (Font, Roma, \& Serra, 2013) to expand the sound palette of the instrument and create backing tracks. The study reported in (Turchet \& Barthet, 2018a) presents an IoMusT ecosystem involving musicians and audiences interacting with the Smart Mandolin, smartphones, and the Freesound repository. The ecosystem was devised to support performer-instrument and performer-audience interactions through the generation of musical accompaniments exploiting crowd-sourced sounds. The authors presented two use cases investigating how audio content retrieved from Freesound can be leveraged by performers or audiences to produce accompanying soundtracks for music performance with a smart mandolin. In the performer-instrument interaction use case, the performer can select content to be retrieved prior to performing through a set of keywords and structure it in order to create the desired accompaniment. In the performer-audience interaction use case, a small group of audience members participated in the music creation by selecting and arranging Freesound audio content to create an accompaniment collaboratively.

\section{User study: Smart guitar as a hub for collaborative music making}

This section describes an ubiquitous smart guitar system where a smart guitar acts as a hub for collaborative music making with a smartphone player. After introducing

\footnotetext{
${ }^{4}$ https://freesound.org/
} 
the system we present a user study to assess the experience of musicians interacting with the system, especially in relation to the potential of SMIs to support ubiquitous music activities.

\subsection{Ubiquitous smart guitar system}

The ubiquitous smart guitar system is composed of a smart guitar based on a conventional acoustic guitar and a smartphone musical app, as described below.

Smart guitar. The smart guitar prototype was based on an electroacoustic nylon string guitar which was enhanced with embedded contact microphone, loudspeaker, low-latency audio processing Bela board (McPherson \& Zappi, 2015), wireless connectivity, and battery. Wireless connectivity was achieved by means of a Wi-Fi USB dongle (A6100-100PES by NETGEAR, which supports the IEEE 802.11ac Wi-Fi standard). Wireless data reception and forwarding were achieved by using OSC messages over the User Datagram Protocol. The audio engine was coded in the Pure Data realtime audio processing environment and comprised a component processing the guitar sound with a simple reverb, and a component responsible for triggering sound samples. The latter consisted of 4 rhythmic loops $(\mathrm{BPM}=150), 4$ drones (with the chords $\mathrm{G}$ min, C min, D maj, G maj), 9 short percussive sounds, and 13 bass sounds ranging from $\mathrm{C} 2$ to $\mathrm{C} 3$, chromatically.

Smartphone musical app. Smartphone apps enabling collaborative musical interactions have often been proposed e.g. for ubiquitous interaction in electronic music ensembles (see e.g. (Barreiro \& Traldi, 2018)). The proposed smartphone musical app uses the TouchOSC environment for iPhone (6S). The app was only used to send OSC messages to the smart guitar, which handled computations and audio processing. The control messages enabled the smartphone user to trigger the sound samples described above and adjust their volume. The app comprised two screen-based user interfaces (see Fig. 3). The first screen (left on Fig. 3) displays the controls for the 4 loops and 4 drones (the first control of each row was used to stop the loop and the drone). The second screen (right on Fig. 3) displays the controls for the drum pads and the 13-note keyboard for the bass.
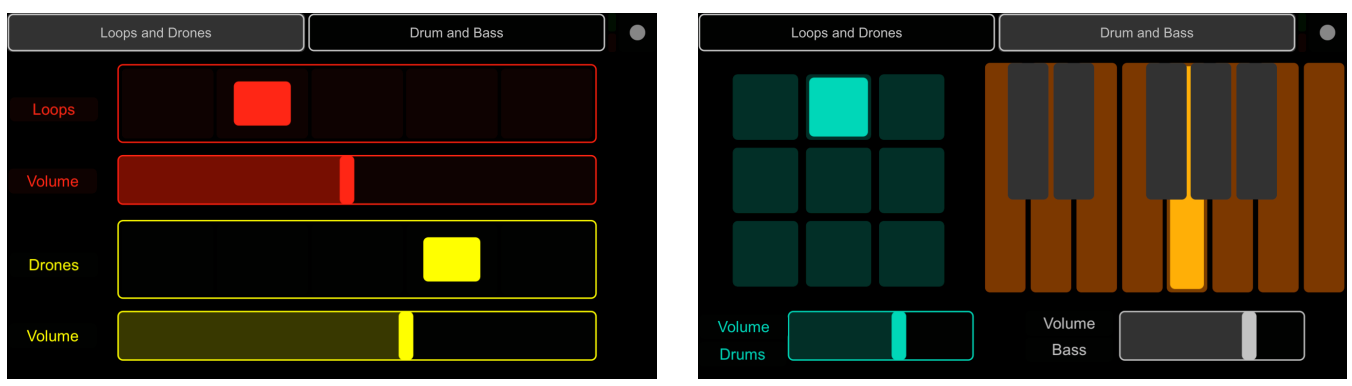

Figure 3. User interface of the smartphone app. UI for the 4 loops and 4 drones (left) and UI for the drum pads and 13-note keyboard for the bass (right).

The system was tested with musicians who had to practice music together.

\subsection{Participants and Setting}

Eighteen participants were recruited, 6 females, 12 males, ranging from 23 to 49 years (average 31.3). Eight participants were guitarists, the other eight had experience as 
electronic music performers. During the study, participants were grouped into pairs with one guitarist and one electronic music performer. None of the participants had previously played with the other, in each pair.

\subsection{Setting and Apparatus}

The study took place in the sound studio of the Centre for Digital Music at Queen Mary University of London. Participants used the ubiquitous smart guitar system described in Section 5.1. Figure 4 shows two participants interacting with the system during the study.

The smart guitar and the smartphone were connected using a Wi-Fi router (TPLink TL-WR902AC) employing the IEEE 802.11ac wireless networking standard over the $5 \mathrm{GHz}$ band. Following the recommendations reported in Mitchell et al. (2014) to optimise the components of a Wi-Fi system for live music performance scenarios to reduce latency and increase throughput, the router was configured in access point mode, security was disabled, and only the IEEE 802.11ac standard was supported.

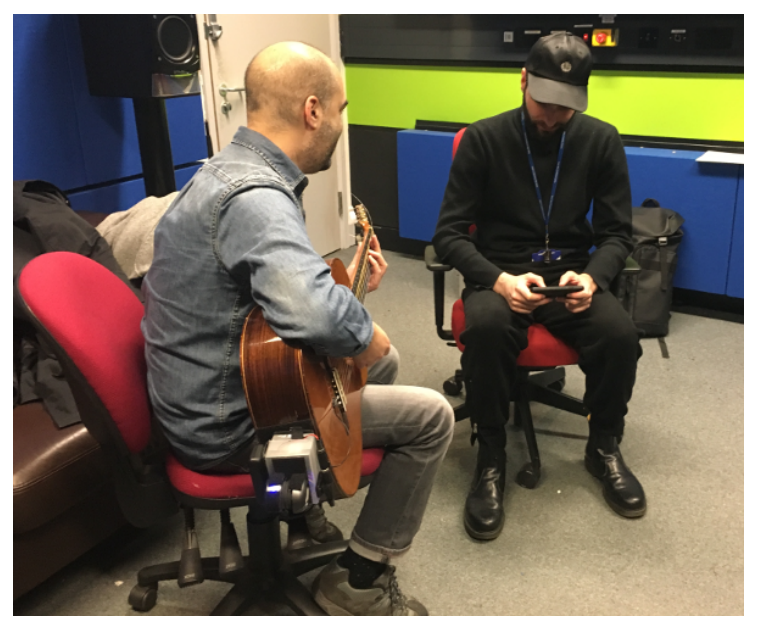

Figure 4. Photo of two participants practicing with the smart guitar and the smartphone app prototypes.

\subsection{Procedure}

For each pair, guitarists were invited to play the smart guitar and electronic music performers, the smartphone. After being introduced to the study, the pair could explore the instruments in a collaborative way for about 10 minutes. After this familiarisation stage, performers were invited to prepare and play three performances of about 5 minutes each. Participants were free to talk to each other to coordinate the performance. After each performance, they were asked to debrief about their musical practice experience using the proposed system. After the three performances, participants had to complete an online questionnaire which is described in the next section. 


\subsection{Survey}

The survey questionnaire was divided into four sections ${ }^{5}$. The first section included demographic questions about gender, age, and musical experience. The second section consisted of the ten System Usability Scale (SUS) questions measured using 5-point Likert items (Brooke et al., 1996). The third section presented the eleven Creativity Support Index (CSI) questions measured using 11-point Likert items (Cherry \& Latulipe, 2014). The CSI section also comprised 15 paired comparisons to determine the relative importance of the six creativity factors in musical practice tasks (Collaboration, Enjoyment, Exploration, Expressiveness, Immersion, Results Worth Effort). The final section of the questionnaire gathered reflective feedback using 10 open-ended questions the topics of which were devised using several human-computer interaction principles. We wanted to find out the hedonic qualities of the system, or lack thereof (What did you like the most about the system?, What did you like the least about the system?). We also asked participants to rate their level of engagement with the system (Bryan-Kinns \& Hamilton, 2012) and the novelty of their experience (two 5-point Likert item questions), and to explain the reasons for their choices (two open-ended questions). The following set of open-ended questions aimed at finding out the possible contexts of use of the system (Would you see yourself using such system and if so in which context(s)?), and how to improve it (In relation to the interface you were using, which types of sounds or backing tracks would you be interested in?, In relation to the interface you were using, which types of audio controls would you be interested in?, How would you improve the system?). Finally, we asked feedback about the participants' experience of the speaker embedded in the smart guitar (How did you feel about hearing all the sounds coming from the smart guitar?), and the added value which they perceived for the system (Which added value do you see in this system?).

\subsection{Results}

We computed the SUS and CSI metrics using Python open-source tools provided at the link below ${ }^{6}$ by customising the code to extract additional parameters and produce figures. We analysed the participant debriefings and open-ended questions using thematic analyses (Braun \& Clarke, 2006).

\subsubsection{System Usability Scale}

The SUS metric assesses the usability of a system on a scale from 0 to 100 . As a point of comparison, an average SUS score of about 68 was obtained from over 500 studies reported at https://measuringu.com/sus/. Figure 5 presents a notched box plot of the SUS scores across participants for the ubiquitous smart guitar system. The system obtained a mean SUS score of 72.4 and median of 72.5 (95\% confidence interval: $[68.9 ; 76.1])$ which is above average. Figure 6 shows the breakdown of the result across the topics of the system usability scale. The results reported in the figure indicates that on overall participants found the system easy to use, simple, quick to learn and to use without technical support. There is however room for improvement to make usability more seamless; users were mildly confident in using the system and unsure of the good integration of its functions and whether they saw themselves using it frequently.

\footnotetext{
${ }^{5}$ We used the online tool https://www.onlinesurveys.ac.uk/

${ }^{6}$ https://github.com/axambo/hci-python-utils
} 


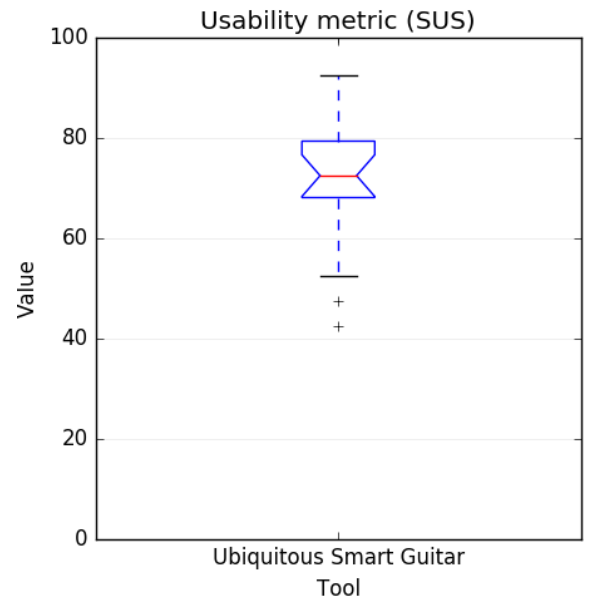

Figure 5. SUS metric for the proposed ubiquitous smart guitar system tested with 18 participants.

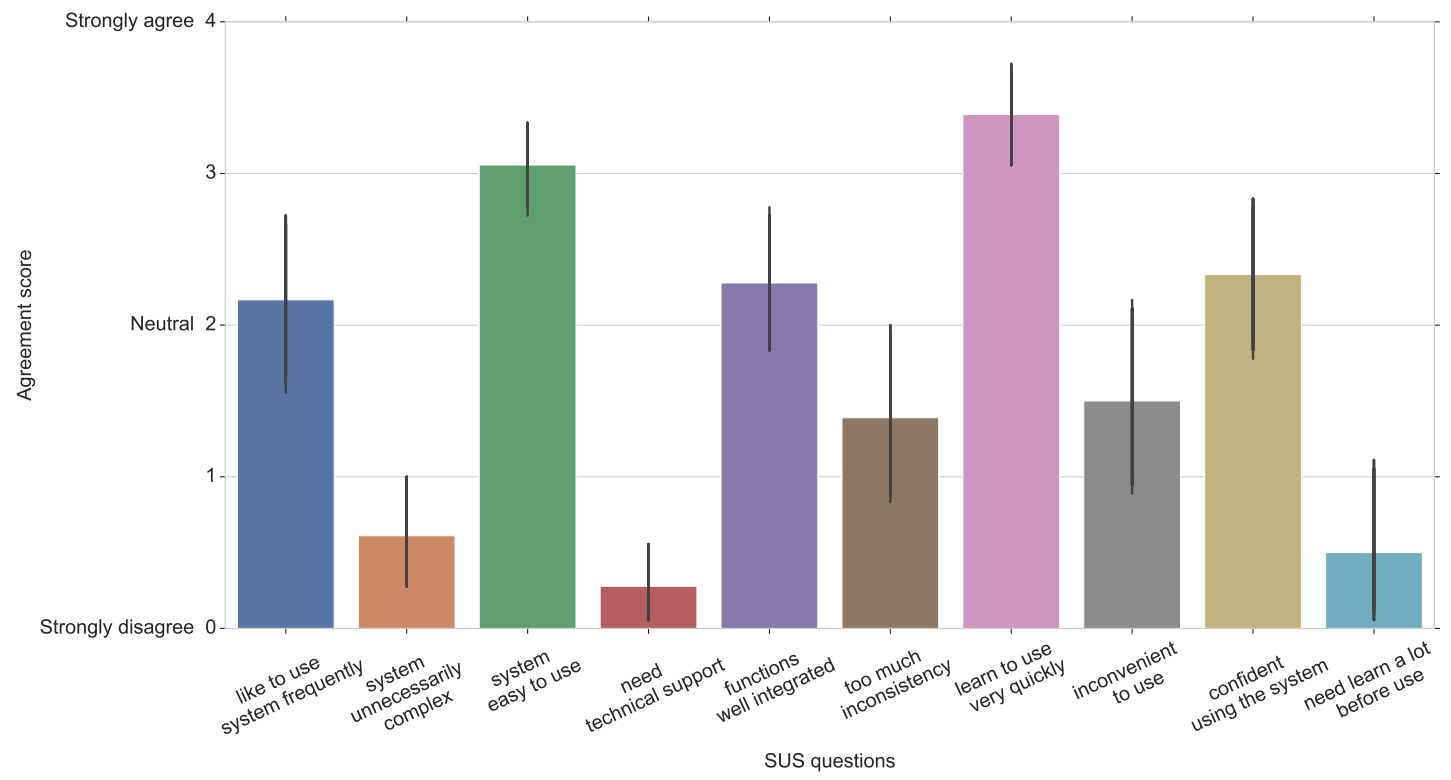

Figure 6. Level of agreement to system usability scale (SUS) topics for the proposed ubiquitous smart guitar system tested with 18 participants. Agreement was measured using 5-point Likert items with 0 corresponding to Strongly disagree and 4 to Strongly agree ( 2 can be considered neutral).

\subsubsection{Creativity Support Index}

The CSI metric enables to assess the ability of a tool to support the open-ended creation of new artefacts (Cherry \& Latulipe, 2014). The ubiquitous smart guitar system obtained a mean CSI of 55.0 and a median CSI of 57.1 (95\% confidence interval: $[48.7 ; 65.7])$ which evidences certain limitations and issues for creativity support. Table 1 presents the average CSI results broke down into factor counts (the number of times a creativity factor was judged more important than another for the task, as based on paired comparisons), factor scores (the ratings of the various factors irrespective of their importance for the task), and the weighted factor scores, which combine the factor counts and scores. The creativity factor which was judged the most important for the collaborative musical practice task is Expressiveness $(\mathrm{M}=3.56 / 5, \mathrm{SD}=1.17)$, 
Table 1. Average CSI results for the collaborative musical practice study with the ubiquitous smart guitar (SD reported in brackets). The highest average value is reported in bold in each column. The mean CSI score is 55.0 ( $\mathrm{SD}=15.6$ ). Ranges: Avg. Factor Counts (0 to 5-i, where $i \in[0 ; 5]$ ), Avg. Factor Score (0 to 10), Avg. Weighted Factor Score (0 to 50-10i, where $i \in[0 ; 5])$. The average weighted factor score is computed from the participants' factor counts and scores (it is not the product between the average factor counts and scores).

\begin{tabular}{|l|c|c|c|}
\hline Creativity factor & $\begin{array}{c}\text { Avg. Factor } \\
\text { Counts }\end{array}$ & $\begin{array}{c}\text { Avg. Factor } \\
\text { Score }\end{array}$ & $\begin{array}{c}\text { Avg. Weighted } \\
\text { Factor Score }\end{array}$ \\
\hline Exploration & $3.33(1.29)$ & $5.31(2.35)$ & $17.19(9.74)$ \\
\hline Expressiveness & $\mathbf{3 . 5 6}(1.17)$ & $5.22(2.36)$ & $\mathbf{1 8 . 5 5}(9.35)$ \\
\hline Immersion & $2.22(1.08)$ & $5.61(2.78)$ & $12.47(9.43)$ \\
\hline Collaboration & $2.78(1.69)$ & $5.53(2.31)$ & $17.47(14.39)$ \\
\hline Enjoyment & $2.17(1.54)$ & $5.86(2.77)$ & $11.44(10.28)$ \\
\hline Results Worth Effort & $0.94(1.08)$ & $\mathbf{6 . 2 8}(1.95)$ & $5.3(7.29)$ \\
\hline
\end{tabular}

closely followed by Exploration $(\mathrm{M}=3.33, \mathrm{SD}=1.29)$. The highest factor score for the system was obtained for the factor Results Worth the Effort $(\mathrm{M}=6.28, \mathrm{SD}=1.95)$ which is probably due to the ease of use previously discussed and the overall satisfying outcome (Enjoyment ranks second with a factor score of $\mathrm{M}=5.86, \mathrm{SD}=2.77$ ). Taking into account the importance of the factors for the task and the factor scores, the highest weighted factor score is obtained for Expressiveness $(\mathrm{M}=18.55, \mathrm{SD}=9.35)$, followed by Exploration $(\mathrm{M}=17.19, \mathrm{SD}=9.74)$. Given that these weighted scores are bounded at 50 and 40 respectively, the results are rather low. The thematic analyses presented in the next section help to understand the creative limitations of the current system and make suggestions for further improvements.

\subsubsection{Engagement and novelty}

The results from the Likert-item questions about engagement with the system and novelty of the experience are shown in Figure 7. The perceived levels of engagement during the activity $(\mathrm{M}=2.83 / 4, \mathrm{SD}=1.17)$ and novelty of the musical experience $(\mathrm{M}=2.56 / 4$, $\mathrm{SD}=1.01$ ) were better than neutral, on average. The thematic analyses presented in the next section bring insights to interpret these results.

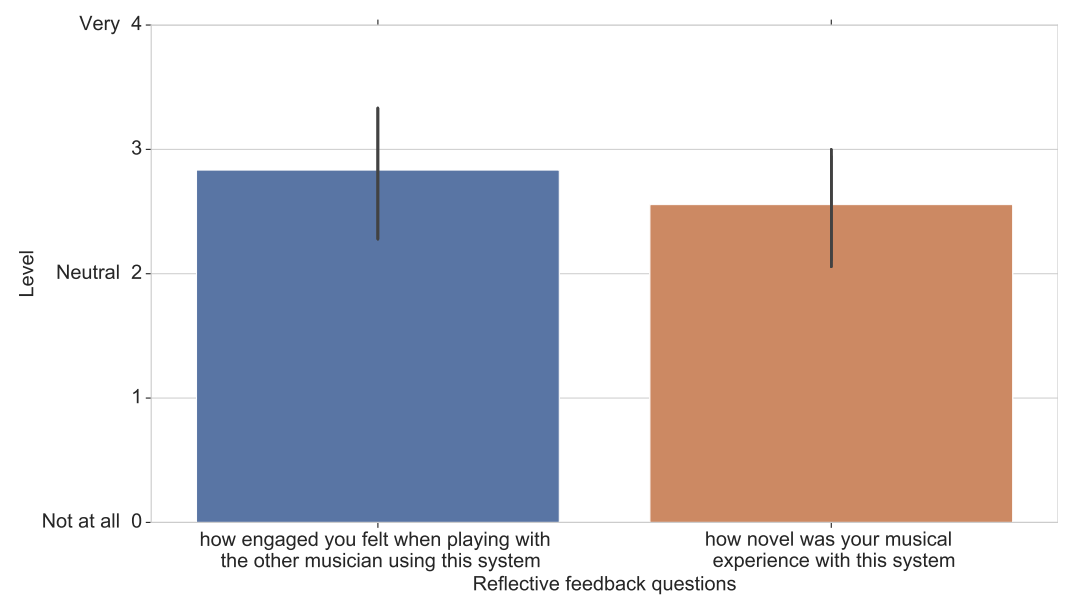

Figure 7. Level of engagement and novelty for the ubiquitous smart guitar tested with 18 participants. Levels were measured using 5 -point Likert items with 0 corresponding to Not at all and 4 to Very ( 2 can be considered neutral). 


\subsubsection{Thematic analyses}

In this section we first present the thematic analysis of the debriefings made by participants between their performances, and then the thematic analysis from the participants' answer to the 10 open-ended questions from the online questionnaire.

5.6.4.1. Thematic analysis of debriefings. The participant debriefings were analysed using an inductive thematic analysis by generating codes from transcripts of the open-ended discussions occurring between participants after each of their performances. The codes were organised into six themes that reflected patterns, as presented below (Issues with face-to-face interaction, Ubiquitous use, Latency and lost controls, Limitations due to size of smartphone UI, Expressive control requests, Customisation).

Issues with face-to-face interaction. Three participants reported that the use of the smartphone app forced them to look at the screen. This was perceived as an obstacle to face-to-face interactions with the guitar player, which could benefit the coordination of the performance. They commented that the smartphone screen did not provide haptic feedback in response to the musicians' actions, and if it did it may enable them to look more at the guitar player.

Ubiquitous use. Three participants reported that the self-containedness of the smart guitar and the possibility to make music with portable devices such as smartphones supports musical activities in a range of settings without complex setups (ubiquity). One participant specifically mentioned the usefulness of this setup compared to situations requiring other digital musical instruments.

Latency and lost controls. Several participants reported to have perceived latency during some interactions, and that a few times their actions on the interface did not result in triggering of the desired sounds. Such issues were due to the current limitations of wireless communication in terms of temporal transmission and network reliability, when using Wi-Fi with the UDP protocol. These issues point towards the need for new ultra-low latency and highly reliable local networks that could be leveraged to enable effective musical interactions. This is one of the challenges discussed for the Internet of Musical Things (IoMusT) paradigm, see (Turchet, Fischione, et al., 2018).

Limitations due to size of smartphone UI. Several smartphone players reported that the size of the smartphone was not optimal for the proposed musical controls. The small size of the phone led these participants to often switch between the two UI screens of the app in order to establish their musical ideas. Moreover, the size of the keyboard controls were deemed too small to allow precise control. On the other hand, most participants judged it empowering to have on the same screen various musical controls (such as the drumpad next to the bass keyboard). Participants suggested the use of a tablet rather than a smartphone for this type of application.

Expressive control requests. On overall, the smartphone players felt that the possibilities offered by the app were too limited in terms of range of possible sounds and expressive controls (Expressiveness is a creative factor judged important for the task). For example, they would have preferred a wider selection of notes for the drones, and more octaves for the keyboard. Moreover, two participants reported that the lack of dynamics of the controlled sounds (which was due to the inability of the screen to track velocity changes associated to different forces applied to it) was a major obstacle for their expressivity. Furthermore, five participants suggested adding the possibility to record the sounds created with the app or with the app and the guitar in order to loop them and create richer textures. 
Customisation. Three participants suggested that the app could be customised to allow the player to choose different layouts for the controls, and to be able to trigger different sound samples of their choice.

5.6.4.2. Thematic analysis of survey open-ended questions. To analyse the survey answers to open-ended questions, we used a hybrid process of inductive and deductive thematic analysis to interpret the raw data (Fereday \& Muir-Cochrane, 2006). The methodological approach integrated theory-driven codes based on the HCI principles described in Section 5.5 and data-driven codes. The 10 open-ended question topics were used as overarching themes. For each of these themes, we uncovered recurring factors from the data, inductively. In total, 682 codes were obtained for the data associated to the 18 participants. These were grouped into the nine following themes (ordered here based on number of code occurrences presented in square brackets): New features [88] (features desired by users), Hedonic - Like the most [31] (what users preferred about the system), Added value [28] (what makes this system enhance existing solutions), Hedonic - Like the least [26] (what users disliked the most about the system), Context of use [24] (which applications were envisioned by users), Limitation [22] (issues with the system), Embedded speaker [14] (participants' experience of the loudspeaker embedded in the smart guitar), Novelty [13] and Engagement [12]. Table 2 reports the results including subthemes and quotes from participants illustrating the themes and codes.

\begin{tabular}{|c|c|}
\hline Themes & Subthemes (bold) and illustrative quotes (italic) \\
\hline \multirow[t]{3}{*}{$\begin{array}{l}\text { New features } \\
(88)\end{array}$} & $\begin{array}{l}\text { Additional audio content (24) - Personalisation of sound } \\
\text { samples and loops: more "pads" and "lead" sounds, more } \\
\text { "organic" and "acoustic" sounds ("less electronic"), more loops } \\
\text { and chord progressions, blues/rock/folk/acoustic/indie pop sounds, } \\
\text { larger range of drum patterns and keys for drones, piano, bass, vi- } \\
\text { olin, percussion sounds, automatic bass lines, backing tracks, con- } \\
\text { trolling guitar tones. }\end{array}$ \\
\hline & $\begin{array}{l}\text { Additional audio controls } \mathbf{( 2 9 )}-\text { Smartphone: loop } \\
\text { pitch/tempo/key/time signature; filters/timbre controls; ADSR } \\
\text { controls; more volume control; sequencer; granular synthesis; haptic } \\
\text { feedback; combining UI screens (backing tracks, pads, keyboards). } \\
\text { Smart guitar: use of electric guitar (tones) and sound fx; volume } \\
\text { control. Both smartphone and smart guitar: recording and } \\
\text { loop control. }\end{array}$ \\
\hline & $\begin{array}{l}\text { Improvement (35) - Smartphone: record and loop; bigger device } \\
\text { (larger keyboard and drum pad), e.g. tablet; octave control; hard- } \\
\text { ware/tactile interface; user-defined sounds; more chords/sounds; fo- } \\
\text { cus on one instrument instead of multiple; ability to process guitar } \\
\text { sound; better audio monitoring; cross-control smartphone/smart } \\
\text { guitar. Smart guitar: more sturdy add-on; minimisation and bet- } \\
\text { ter integration; looping functionality; change of guitar tones. }\end{array}$ \\
\hline
\end{tabular}




\begin{tabular}{|c|c|}
\hline $\begin{array}{l}\text { Hedonic - } \\
\text { Like the } \\
\text { most (31) }\end{array}$ & $\begin{array}{l}\text { Fun: "fun to use"; sound intimacy and connection: "I did like } \\
\text { the proximity of the audio and if I was going to be in a situation } \\
\text { where I was playing electronic instruments, would be up for that as } \\
\text { an audio option", "I liked feeling the synth bass response within the } \\
\text { body of the guitar", "The smart phone sounds coming out of the } \\
\text { guitar is an interesting and mostly positive feature as I feel more } \\
\text { "connected" to the performance.", "The speaker system is mounted } \\
\text { to the guitar which makes it somewhat "part of the instrument", } \\
\text { which possibly gets the guitarist more involved with the sound; Tim- } \\
\text { bre accessibility and simplicity of use: "I particularly liked the } \\
\text { function of the app allowing to create a drone/pad and then this } \\
\text { would loop. I also liked the bass sounds, which had a good timbre. } \\
\text { In terms of the design I thought it was very simple and easy to navi- } \\
\text { gate - if you have used a DAW or something similar before."; Band } \\
\text { augmentation: "The smart phone system allows for more textured } \\
\text { / layered sound to be produced such that two people can perform the } \\
\text { same music that would take a larger ensemble", "I liked that the app } \\
\text { managed to create the presence of multiple virtual musicians (eg. } \\
\text { drums and keyboards)"; New musical practice: "It did present a } \\
\text { new kind of musical accompaniment/soloist relationship."; Ubiq- } \\
\text { uity: "Small size, portability"; Acoustic/digital blend: "The sys- } \\
\text { tem allows real-time interaction between musicians with acoustic } \\
\text { instruments and digital instruments". UI affordances: "keyboard } \\
\text { and drum pads beside one another" }\end{array}$ \\
\hline $\begin{array}{l}\text { Added value } \\
(28)\end{array}$ & $\begin{array}{l}\text { Ubiquity: "Easiness of the setup" and "portability, "Sketching } \\
\text { ideas on the go", "I think it is portable and could be used for } \\
\text { writing on the move, without have to open a laptop etc.", Inclu- } \\
\text { siveness: "Potentially allows non-musicians to engage informally } \\
\text { with guitarists in an immediate and unifying way.", "for people who } \\
\text { don't play an instrument"; Novel practices: improvisation, jam- } \\
\text { ming, interaction with other musicians, real-time interaction be- } \\
\text { tween acoustic instruments and DMIs ("audio blending"); Peda- } \\
\text { gogy: "using it for learning guitar, using the tool as interactive } \\
\text { accompaniment", "for guitar teachers". }\end{array}$ \\
\hline $\begin{array}{l}\text { Hedonic - } \\
\text { Like the } \\
\text { least }(26)\end{array}$ & $\begin{array}{l}\text { Smartphone UI too small; Lack of personalisation of sounds } \\
\text { and expressive controls: "I didn't like the sound sets, and felt } \\
\text { that my partner had limited options for jamming with me.", "I had } \\
\text { no control on the sounds; Buzzing (loudspeaker); Non homoge- } \\
\text { nous dynamics: "The volumes of the samples were not matching } \\
\text { much, some were louder, some quiet, so to play more than one, one } \\
\text { would need to change the volume"; Lack of recording/looping } \\
\text { functions. Tuning issue between app and smart guitar; Lack of } \\
\text { responsiveness (timing and haptic feedback). }\end{array}$ \\
\hline
\end{tabular}




\begin{tabular}{|c|c|}
\hline $\begin{array}{l}\text { Context of } \\
\text { use }(24)\end{array}$ & $\begin{array}{l}\text { For non musicians/beginners: "I think this system adds the } \\
\text { most amount of value when targeted to beginner or non-musicians } \\
\text { as it opens up a way for those people to collaborate and engage with } \\
\text { the music-making process."; Pedagogy "to learn guitar, using the } \\
\text { tool as interactive accompaniment", "teachers could use it for their } \\
\text { students as a challenge to play along"; Live or improvisation; } \\
\text { Ubiquitous use: "for fun with friends", "casual, social situations } \\
\text { or impromptu collaborative performance", "home reharsing or play- } \\
\text { ing with friends, "when I am travelling and want to make music"; } \\
\text { Composition: as a "writing tool". }\end{array}$ \\
\hline $\begin{array}{l}\text { Limitation } \\
(22)\end{array}$ & $\begin{array}{l}\text { Technology barrier to natural performer communication: } \\
\text { "The nature of a touch screen meant the smart phone player was } \\
\text { constantly looking down to focus on playing the right notes or ex- } \\
\text { ploring the application. This takes the performer "out" of the im- } \\
\text { provisation and makes it feel a lot more like I am performing with } \\
\text { a computer than another human"; Lack of expressiveness: e.g. } \\
\text { need a larger choice of sounds, will to use electric guitar and gui- } \\
\text { tar fx, keys too restricted; UI too small: "This actually made it } \\
\text { very difficult to play both the drums and the bass at the same time } \\
\text { because it was difficult for me to accurately hit the pads and notes } \\
\text { that I was aiming for. This made it unpredictable as to whether I } \\
\text { would play the notes that I was intending."; Latency: "Latency on } \\
\text { the bass was distracting. I think on the drums, playing repeatedly, I } \\
\text { adjust my timing to get the sound to match - something I'm used to } \\
\text { doing as playing gamelan, I place my sound rather than my action. } \\
\text { On the bass, I wasn't repeating as much and so was more thrown by } \\
\text { the latency."; Lack of clarity of the mixed audio: "Generally } \\
\text { it's fine but sometimes the mix gets confusing when sounds fall into } \\
\text { the same register (masking effects)", "There are certain sounds that } \\
\text { distorted which sounded odd." }\end{array}$ \\
\hline $\begin{array}{l}\text { Embedded } \\
\text { speaker (14) }\end{array}$ & $\begin{array}{l}\text { Positive emotional engagement: "fun", "great idea", "enjoyed"; } \\
\text { Immersion: "I enjoyed this from a "selfish" level. Although it felt } \\
\text { less like I was collaborating with another musician, it was nice to } \\
\text { be able to clearly hear the output of the system without worrying } \\
\text { about where I or the other performer are in the space"; Spatial } \\
\text { location issue: "although from my position I perhaps didn't get } \\
\text { the full benefit. Also it was a little difficult at points to hear some of } \\
\text { the parts."; Volume control issue: "I felt I couldn't control them } \\
\text { well since they were louder for the guitarist than myself.". }\end{array}$ \\
\hline
\end{tabular}




\begin{tabular}{|l|l|}
\hline Novelty (13) & Serendipity: "This resulted in 'happy accidents' and also resulted \\
in different patterns and notes than I usually would play if given \\
other interfaces (such as a physical USB midi keyboard, or a larger \\
drum pad that was not on an iphone gui)"; Novel musical prac- \\
tice and experience: "We jammed on known chord progressions \\
and riffs so the music wasn't novel, but the interface meant I person- \\
ally had a different experience.", "I never performed with someone \\
playing an instrument able to transduce both my interpretation and \\
his interpretation", "novelty was having it attached to the guitar, \\
and actually using it to play along with someone rather than using \\
it with a DAW"; Immersive sound: "The bass response in the \\
body of the guitar was very unusual", "There is something uniquely \\
unifying about the affect of having a digital controller influence the \\
sound being generated from the body of the guitar. For example, \\
feeling the vibration of the bass synth whilst playing: that presented \\
the kind of immediate connection and sensation that you only get \\
when performing with a full band in rehearsal or on stage. It brings \\
a sense of synergy that would otherwise be difficult to achieve in \\
casual situations." \\
$\begin{array}{ll}\text { Lack of naturalness: "It was fun but it didn't feel quite like play- } \\
\text { ing with another musician (drummer or bassist)"; Lack of expres- } \\
\text { sivity: "I felt there was only moderate engagement with the other } \\
\text { musician as the tool was dictating my expressivity"; Flow: "This } \\
\text { interface required constant input so it was very engaging. It wasn't } \\
\text { like I would just press play on a loop and leave it at that. I would } \\
\text { want to add to it - such as turning on and off the drones, playing } \\
\text { the drum pads and playing the bass sounds.", "We were completely } \\
\text { focused on doing something that sounded good", "I felt we were able } \\
\text { to get hooked on some interesting musical ideas forgetting about the } \\
\text { technology"; Ease of collaboration: 'It was easy to collaborate } \\
\text { with the other person"; Learning curve: "I think to maintain a } \\
\text { more engaged feeling, both musicians should have similar expertise } \\
\text { on their instrument. as the digital interface was introduced as a new } \\
\text { instrument, the musicians need time to master on it." }\end{array}$ \\
\hline (12)
\end{tabular}

Table 2.: Themes, subthemes and illustrative quotes for the thematic analysis of 10 open-ended questions from 18 participants. The order of the themes is based on the number of code occurrences which are reported in brackets.

\subsection{Discussion}

The quantitative and qualitative results presented in this section shows that, on average, participants found the ubiquitous smart guitar system easy to use (high SUS score) and judged their experience during their musical practice to be "fun" (thematic analysis). Overall, participants felt engaged during the activity and found their interaction with the other musician novel to some extent. This can be related to the "ease" with which the system let them collaborate with one another, and the possibility to augment the ensemble by creating the "presence of multiple virtual musicians" 
with the musical app. For some, the novelty came from the blending of acousticallyand digitally-generated sounds on one of the instruments creating a sense of intimacy and intrinsic connection between performers. Furthermore, this facilitated "unusual" collaborations between performers of acoustic and digital music instruments. Several participants described situations of flow being engaged in the musical activity exploring and exploiting the different affordances of the musical app while keeping the focus to mutually create a performance with the other musician. However, some participants felt a lack of musical naturalness (e.g. bass, drum) compared to more common performances with an ensemble, and felt restricted by the smartphone musical app which was "dictating their expressivity". Another factor limiting engagement for the smartphone users was the need to learn how the interface of the musical app worked, compared to guitarists which already knew how to play their instrument.

Strong support was found for the ubiquitous quality of the system which was the main design motivation. Participants highlighted the "small size", "portability" and "ease of setup" of the system. They expressed its suitability for jamming, rehearsing or composing in mundane contexts such as home, social situations (with friends), or travelling ("sketching ideas on the go"). The system was also commended for its inclusiveness by enabling non musicians to play with guitarists. The configurable background accompaniments and embedded speaker were also judged promising for musical pedagogy, whether for self-learning or guitar teachers. The speaker embedded on the smart guitar sparked positive emotional engagement ("fun", "great", "enjoyed") and fostered immersion in the performance but several issues emerged towards volume control, spatial location and audio mixing.

The study also enabled to shed light on several frictions that hindered creativity support and engagement. The expressive affordances of the smartphone musical app were judged limited for several reasons: (i) the smartphone UI was judged too small for some of the instruments which were implemented (e.g. keyboard), (ii) lack of personalisation (some participants didn't like the sound presets, and not having enough control on the sounds or the chord progressions, and wished to be able to use their own sounds), (iii) homogeneity and quality of sound content (some participants found that the sounds did not match well, and that their dynamics was not homogenous), (iii) the lack of recording and looping functionality, and (iv) tuning issues (when playing along with the guitar). Several issues related to responsiveness were also reported due to latency effects and the lack of haptic feedback when playing the smartphone app. The smartphone app, which constricted the attention of one of the musicians to a small screen, was also perceived by some as a barrier for co-performer communication.

The participants made several suggestions to improve the ubiquitous smart guitar system. Smartphone users commonly wished to have at disposal a larger UI (e.g. through a tablet), and to be able to personalise and curate the sound content proposed by the app (using their own audio content, or user-defined sounds through synthesis and audio processing). Both smartphone and smart guitar users required more expressive controls (e.g. synthesis, audio effects) with recording and looping capabilities. The responsiveness of the system should also be improved by reducing latency and addressing the lack of haptic feedback of the smartphone musical app, common in DMIs. Some participants were interested in the cross-control capabilities of the system and wanted more agency to act on what the other musician was playing based on their own performance. At the practical level, smart guitar users also wished for a better integration of the guitar "add-on". 


\section{Overall Discussion}

This paper explored how smart musical instruments could be applied to ubiquitous musical activities. To date, UbiMus activities have mostly involved mobile devices such as smartphones or custom-built devices according to do-it-yourself practices typical of the maker community (Brown, Keller, \& de Lima, 2018; Keller et al., 2014; Lazzarini, Keller, \& Pimenta, 2015). In this paper, the authors have attempted to provide arguments showing how SMIs can support ubiquitous musical activities. In Section 3, we presented three SMIs features (self-contained nature, connectivity and embedded intelligence) deemed to be well suited for ubiquitous musical activities.

The UbiMus research community has so far mostly targeted creative practices involving non-professional musicians, and focused a great part of its vision on accessibility aspects (Brown et al., 2018). In a complementary way, SMIs enable UbiMus activities that can target professional performers using augmented versions of conventional instruments. Although SMIs are not ubiquitous yet, they can be used in conjunction with ubiquitous technologies such as smartphones. Contrary to mobile devices such as smartphones, SMIs based on traditional instruments benefit from the improvements made to the instruments over the years through lutherie and which provide musicians with great control intimacy, an aspect often limited in current digital music interfaces (Wessel \& Wright, 2002).

To date, only a handful of SMIs have been developed in industrial contexts and only a little academic research has been conducted in this area. This implies that SMI-based UbiMus activities have been less widespread compared to other approaches relying on smartphones. Interesting use cases for SMIs can be envisioned in UbiMus contexts such as technology-mediated audience participation (Hödl, Fitzpatrick, \& Kayali, 2017; Wu et al., 2017). For instance, SMIs could be used to create performer-audience interactions by letting the audience produce accompaniment according to musical information sent from SMIs to connected smartphones. It would also be interesting to investigate how SMIs could be used in conjunction with new interfaces for musical expression supporting body movement and hand gesture inputs enabling embodied IoMusT interactions (see e.g. (Keller, Gomes, \& Aliel, 2018)).

\section{Conclusion}

This paper investigated how the smart musical instrument paradigm could be applied to ubiquitous music from a theoretical perspective and through a use case involving an ubiquitous smart guitar system. The proposed system enables a guitarist and a smartphone user to practice music together using a smart guitar as a hub for sound reproduction. Results from a user study conducted with 18 performers evidenced that the system holds ubiquitous qualities such as ease of use, portability and inclusiveness. Several contexts of use were envisioned by participants ranging from casual social musical interactions to composing on the go and musical pedagogy. By blending both performers' sonic actions into a single audio source through an embedded speaker, the system also enabled novel musical immersion and connection between acoustic and digital music instrumentalists. Feedback from smartphone users highlighted the need for larger user interface for certain musical interactions (e.g. musical keyboard) and the desire to personalise and craft their own audio content rather than using predefined samples. Latency (network and audio processing) hindered the responsiveness of the system in some cases and the lack of haptic feedback of the smartphone musical app 
reduced understanding of actions and control intimacy, an aspect common in DMIs. Interestingly, some participants wished to explore more the notion of cross-control between performers, a feature that could be the object of future SMI research.

Several challenges remain to be solved to enable the seamless integration of SMI technology in UbiMus activities including interoperability, latency, the development of intelligent services using Artificial Intelligence, and the miniaturization and integration of embedded systems. It is the authors' hope that this work can stimulate further discussions on this topic and that researchers and practitioners in the two fields can benefit from the empirical results presented in this paper to develop new SMIs supporting UbiMus activities.

\section{Funding}

We acknowledge support from the EU H2020 Marie Curie Individual Fellowship 'Towards the Internet of Musical Things' (749561) and the EU H2020 grant 'The Audio Commons Initiative' (688382). We also would like to thank the participants who took part in the study for their time and valuable feedback.

\section{References}

Barreiro, D., \& Traldi, C. (2018). Musical conceptions and strategies in creative activities with mobile devices. In Proceedings of the Eight Workshop on Ubiquitous Music (p. 132-143).

Berdahl, E. (2014). How to make embedded acoustic instruments. In Proceedings of the Conference on New Interfaces for Musical Expression (pp. 140-143).

Bødker, S. (2015). Third-wave HCI, 10 Years Later-participation and Sharing. Interactions, 22(5), 24-31. Retrieved from http://doi.acm.org/10.1145/2804405

Borgia, E. (2014). The Internet of Things vision: Key features, applications and open issues. Computer Communications, 54, 1-31.

Braun, V., \& Clarke, V. (2006). Using thematic analysis in psychology. Qualitative Research in Psychology, 3(2), 77-101.

Brooke, J., et al. (1996). Sus-a quick and dirty usability scale. Usability evaluation in industry, $189(194), 4-7$.

Brown, A. R., Keller, D., \& de Lima, M. H. (2018). How ubiquitous technologies support ubiquitous music. The Oxford Handbook of Community Music, 131-151.

Bryan-Kinns, N., \& Hamilton, F. (2012). Identifying mutual engagement. Behaviour 8 Information Technology, 31(2), 101-125.

Burgoyne, J., Fujinaga, I., \& Downie, J. (2016). Music information retrieval. A New Companion to Digital Humanities, 213-228.

Cherry, E., \& Latulipe, C. (2014). Quantifying the creativity support of digital tools through the creativity support index. ACM Transactions on Computer-Human Interaction, 21(4), 21.

Dannenberg, R., \& Hu, N. (2003). Pattern discovery techniques for music audio. Journal of New Music Research, 32(2), 153-163. Retrieved from https://doi.org/10.1076/jnmr .32 .2 .153 .16738

Fereday, J., \& Muir-Cochrane, E. (2006). Demonstrating rigor using thematic analysis: A hybrid approach of inductive and deductive coding and theme development. International journal of qualitative methods, 5(1), 80-92.

Fiebrink, R., \& Caramiaux, B. (2016). The machine learning algorithm as creative musical tool. In R. Dean \& A. McLean (Eds.), Oxford handbook of algorithmic music. Oxford University Press. 
Font, F., Brookes, T., Fazekas, G., Guerber, M., La Burthe, A., Plans, D., .. Serra, X. (2016). Audio commons: bringing creative commons audio content to the creative industries. In Audio engineering society conference: 61st international conference: Audio for games.

Font, F., Roma, G., \& Serra, X. (2013). Freesound technical demo. In Proceedings of the ACM international conference on Multimedia (pp. 411-412).

Hödl, O., Fitzpatrick, G., \& Kayali, F. (2017). Design implications for technology-mediated audience participation in live music. In Proceedings of the sound and music computing conference (pp. 28-34).

Jensenius, A., \& Lyons, M. (2017). A nime reader: Fifteen years of new interfaces for musical expression. Springer. Retrieved from https://doi.org/10.1007/978-3-319-47214-0

Keller, D., Gomes, C., \& Aliel, L. (2018). The Handy Metaphor: Bimanual, touchless interaction for the Internet of Musical Things. In Proceedings of the Eight Workshop on Ubiquitous Music (pp. 180-188).

Keller, D., \& Lazzarini, V. (2017). Ecologically grounded creative practices in ubiquitous music. Organised Sound, 22(1), 61-72. Retrieved from https://doi.org/10.1017/ S1355771816000340

Keller, D., \& Lazzarini, V. (2018). Theoretical approaches to musical creativity: The ubimus perspective. Musica Theorica, 2(1).

Keller, D., Lazzarini, V., \& Pimenta, M. (2014). Ubiquitous music. Springer.

Lazzarini, V., Keller, D., \& Pimenta, M. S. (2015). Prototyping of ubiquitous music ecosystems. Journal of Cases on Information Technology, 17(4), 73-85.

MacConnell, D., Trail, S., Tzanetakis, G., Driessen, P., Page, W., \& Wellington, N. (2013). Reconfigurable autonomous novel guitar effects (RANGE). In Proceedings of the international conference on sound and music computing.

Martinez-Avila, J., Greenhalgh, C., Hazzard, A., Benford, S., \& Chamberlain, A. (2019). Encumbered interaction: a study of musicians preparing to perform. In Proceedings of the conference on human factors in computing systems.

McPherson, A. (2015). Buttons, handles, and keys: Advances in continuous-control keyboard instruments. Computer Music Journal, 39(2), 28-46. Retrieved from https://doi.org/ 10.1162/COMJ_a_00297

McPherson, A., Jack, R., \& Moro, G. (2016). Action-sound latency: Are our tools fast enough? In Proceedings of the Conference on New Interfaces for Musical Expression.

McPherson, A., \& Zappi, V. (2015). An environment for Submillisecond-Latency audio and sensor processing on BeagleBone black. In Audio engineering society convention 138. Audio Engineering Society. Retrieved from http://www.aes.org/e-lib/browse.cfm?elib= 17755

Miranda, E., \& Wanderley, M. (2006). New digital musical instruments: control and interaction beyond the keyboard (Vol. 21). AR Editions, Inc.

Mitchell, T., Madgwick, S., Rankine, S., Hilton, G., Freed, A., \& Nix, A. (2014). Making the most of wi-fi: Optimisations for robust wireless live music performance. In Proceedings of the Conference on New Interfaces for Musical Expression (pp. 251-256).

Müllensiefen, D., Gingras, B., Musil, J., \& Stewart, L. (2014). The musicality of non-musicians: an index for assessing musical sophistication in the general population. PloS one, 9(2), e89642.

Overholt, D., Berdahl, E., \& Hamilton, R. (2011). Advancements in actuated musical instruments. Organised Sound, 16(02), 154-165. Retrieved from https://doi.org/10.1017/ S1355771811000100

Pardue, L., Harte, C., \& McPherson, A. (2015). A low-cost real-time tracking system for violin. Journal of New Music Research, 44 (4), 305-323. Retrieved from https://doi .org/ $10.1080 / 09298215.2015 .1087575$

Rossitto, C., Rostami, A., Tholander, J., McMillan, D., Barkhuus, L., Fischione, C., \& Turchet, L. (2018). Musicians' initial encounters with a smart guitar. In Proceedings of the 10th nordic conference on human-computer interaction (pp. 13-24). New York, NY, USA: ACM. Retrieved from http://doi.acm.org/10.1145/3240167.3240223 
Rottondi, C., Chafe, C., Allocchio, C., \& Sarti, A. (2016). An overview on networked music performance technologies. IEEE Access, 4, 8823-8843. Retrieved from https://doi.org/ 10.1109/ACCESS . 2016.2628440

Rowland, C., Goodman, E., Charlier, M., Light, A., \& Lui, A. (2015). Designing connected products: Ux for the consumer internet of things. O'Reilly Media, Inc.

Satyanarayanan, M. (2001). Pervasive computing: Vision and challenges. IEEE Personal communications, 8(4), 10-17.

Schiavoni, F., de Faria, P., \& Manzolli, J. (2018). Addressing Creativity in Network Communication for Computer Music Interaction. In Proceedings of the Eight Workshop on Ubiquitous Music (pp. 144-156).

Slaney, M. (2002). Semantic-audio retrieval. In Ieee international conference on acoustics, speech, and signal processing (Vol. 4, pp. 4108-4111). Retrieved from https://doi.org/ 10.1109/ICASSP. 2002.5745561

Stolfi, A., Ceriani, M., Turchet, L., \& Barthet, M. (2018). Playsound.space: Inclusive Free Music Improvisations Using Audio Commons. In Proceedings of the Conference on New Interfaces for Musical Expression (pp. 228-233).

Turchet, L. (2018a). Smart Mandolin: autobiographical design, implementation, use cases, and lessons learned. In Proceedings of Audio Mostly Conference (pp. 13:1-13:7). Retrieved from http://doi.acm.org/10.1145/3243274.3243280

Turchet, L. (2018b). Some reflections on the relation between augmented and smart musical instruments. In Proceedings of Audio Mostly Conference (pp. 17:1-17:7). Retrieved from http://doi.acm.org/10.1145/3243274.3243281

Turchet, L. (2019). Smart Musical Instruments: vision, design principles, and future directions. IEEE Access, 7, 8944-8963. Retrieved from https://doi.org/10.1109/ACCESS .2018. 2876891

Turchet, L., \& Barthet, M. (2018a). Jamming with a smart mandolin and Freesound-based accompaniment. In IEEE Conference of Open Innovations Association (FRUCT) (pp. 375381).

Turchet, L., \& Barthet, M. (2018b). Ubiquitous musical activities with smart musical instruments. In Proceedings of the Eight Workshop on Ubiquitous Music.

Turchet, L., \& Barthet, M. (2019). Co-design of Musical Haptic Wearables for electronic music performer's communication. IEEE Transactions on Human-Machine Systems, 49(2), 183193.

Turchet, L., Benincaso, M., \& Fischione, C. (2017). Examples of use cases with smart instruments. In Proceedings of Audio Mostly Conference (pp. 47:1-47:5). Retrieved from https://doi.org/10.1145/3123514.3123553

Turchet, L., Fischione, C., Essl, G., Keller, D., \& Barthet, M. (2018). Internet of Musical Things: Vision and Challenges. IEEE Access, 6, 61994-62017.

Turchet, L., McPherson, A., \& Barthet, M. (2018a). Co-design of a Smart Cajón. Journal of the Audio Engineering Society, 66(4), 220-230. Retrieved from https://doi.org/10.17743/ jaes. 2018.0007

Turchet, L., McPherson, A., \& Barthet, M. (2018b). Real-time hit classification in a Smart Cajón. Frontiers in ICT, 5(16). Retrieved from https://doi.org/10.3389/fict.2018 .00016

Weiser, M. (1991). The computer for the 21st century. Scientific american, 265(3), 94-105.

Wessel, D., \& Wright, M. (2002). Problems and prospects for intimate musical control of computers. Computer music journal, 26(3), 11-22. Retrieved from https://doi.org/ 10.1162/014892602320582945

Wu, Y., Zhang, L., Bryan-Kinns, N., \& Barthet, M. (2017). Open symphony: Creative participation for audiences of live music performances. IEEE MultiMedia, 24 (1), 48-62. 